\title{
Efficient high NA flat micro-lenses realized using high contrast transmitarrays
}

\author{
Amir Arbabi ${ }^{1 *}$, Yu Horie ${ }^{1}$, Alexander J. Ball ${ }^{1}$, Mahmood Bagheri ${ }^{2}$, Andrei Faraon ${ }^{1}$ \\ ${ }^{1}$ T. J. Watson Laboratory of Applied Physics, California Institute of Technology, 1200 E California \\ Blvd., Pasadena, CA 91125 \\ ${ }^{2}$ Jet Propulsion Laboratory, California Institute of Technology, Pasadena, CA 91109, USA \\ *amir@caltech.edu
}

\begin{abstract}
We present design, fabrication, and characterization results of high numerical aperture (NA) micro-lenses based on a high contrast transmitarray platform. The high contrast transmitarray is created by periodic arrangement of amorphous silicon posts with different diameters on a fused silica substrate. We report near infrared high NA micro-lenses with spot sizes as small as $0.57 \lambda$ and focusing efficiencies in excess of $80 \%$. We demonstrate a trade-off relation between NA and efficiency of high contrast array flat micro-lenses, and attribute it to the spatial discretization of their phase profiles.

Keywords: Flat micro-lens, high numerical aperture, metasurface, transmitarray, high index contrast, grating, diffractive lens
\end{abstract}

\section{INTRODUCTION}

Flat micro-lenses with high numerical apertures (NAs) that focus the light to small spot sizes are attractive in many applications including data recording and retrieval, high speed detectors, and integrated micro-optical systems. Diffractive flat micro-lenses are essentially phase masks which shape optical wavefronts. Several approaches based on multilevel Fresnel structures [1-7], effective medium structures [8-12], plasmonic metasurfaces [13-19], and high contrast aperiodic gratings [20-23] have been used for achieving wavefront control. Multilevel Fresnel structures approximate a continuous thickness variation corresponding to a desired phase profile, by a limited number of steps. Fresnel micro-lenses with full width at half maximum (FWHM) focal spot sizes as small as $\lambda$ corresponding to an NA of 0.5 have been reported [24]. One drawback of multilevel structures is the fabrication complexity involving gray-scale lithography. Another approach for controlling optical wavefronts relies on using aperiodic gratings patterned with deep subwavelength features that operate in the effective medium regime. Diffractive components such as blazed gratings [10, 12] and micro-lenses [11] have been realized using this approach. The required deep subwavelength feature sizes and very high aspect ratio structures have limited the practical applications of this technique. Recently, diffractive optical elements based on plasmonic metasurfaces have attracted significant attention $[25,26]$. Diffractive components such as micro-lenses [13, 17, 18], axicons [17], and complex beam shapers [16, 19] have been implemented; however, they suffer from fundamental limitations [27, 28] and material optical absorption losses, which have limited the efficiency of a these devices to a few percent [17].

Dielectric diffractive elements based on aperiodic 1D high contrast gratings (HCGs) have achieved higher efficiencies [29][20, 21, 30] . Cylindrical focusing mirrors have been realized by gradually modifying the local period and duty cycle of reflective HCGs [20,21]. Using a similar 1D grating structure and by changing the duty cycle and period along the grating bars, a polarization sensitive spherical mirror with the measured airy disk diameter spot size of approximately $5 \lambda$ (corresponding to NA of 0.24 ) has been reported [23]. Due to the strong coupling along the grating lines, high NA micro-lenses, which have rapidly varying phase profiles, have not been successfully implemented using 1D HCGs. Two dimensional high contrast gratings have been recently used to implement low NA micro-lenses [31, 32], focusing mirrors, and retroreflectors [33]. Here, by experimental demonstration of efficient high NA micro-lenses, we show that a special class of two dimensional high contrast grating, which we refer to as high contrast arrays, provide both large efficiency and high spatial resolution required for sampling the phase profiles of a various flat diffractive elements. We first discuss the properties of high contrast arrays, and then present design, fabrication and characterization details of HCTA high NA micro-lenses.

High Contrast Metastructures IV, edited by Connie J. Chang-Hasnain, David Fattal,

Fumio Koyama, Weimin Zhou, Proc. of SPIE Vol. 9372, 93720P · ๔ 2015 SPIE

CCC code: $0277-786 \mathrm{X} / 15 / \$ 18 \cdot$ doi: $10.1117 / 12.2079509$

Proc. of SPIE Vol. 9372 93720P-1 
(a)

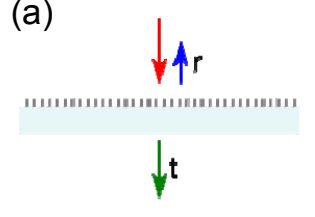

0 -

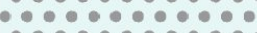
$\therefore \circ$ :  $\because$

(c)

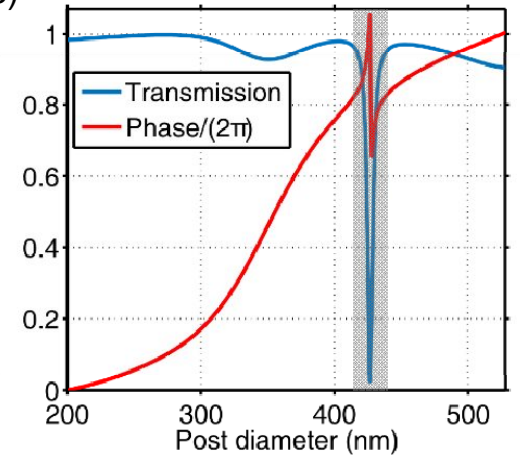

(b)

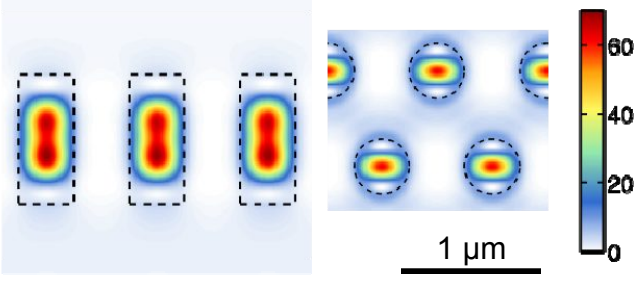

(d)

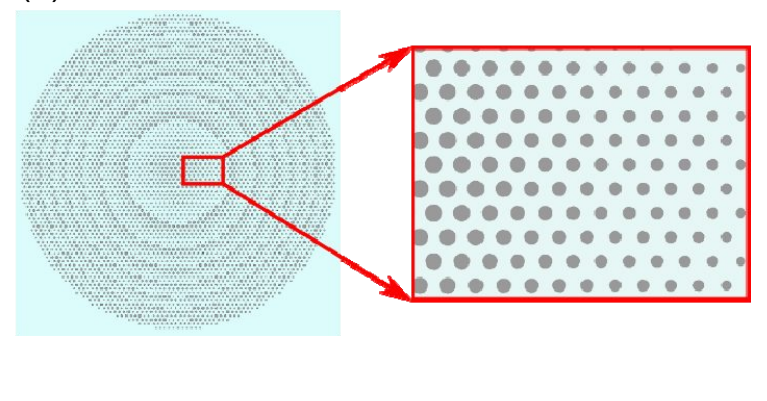

Figure 1. (a) Schematic illustration of a periodic high contrast transmitarray composed of circular aSi posts on a glass substrate which are arranged in a triangular lattice. The optical response of the grating is characterized by its reflection and transmission coefficients. (b) Top and side views of the color coded magnetic energy density for the grating shown in (a). A plane wave with magnetic energy density if 1 is incident from top. (c) Transmission and the phase of the transmission coefficient of the high contrast transmitarray shown in (a) as a function of the diameter of the posts. The shaded region indicates post diameter values which were excluded in the design of micro-lenses. (d) Schematic illustration of an aperiodic high contrast array which implements a micro-lens. The expanded view shows the gradual variation of the post diameters while the lattice is unchanged.

\section{HIGH CONTRAST TRANSMITARRRAYS MICRO-LENSES}

A zeroth order grating is a 2D periodic array of scatterers with a subwavelength period. An example of such a grating made of disconnected scatterers is shown in Fig. 1 (a). The grating is composed of circular aSi posts which are located on a fused silica substrate and arranged in a hexagonal lattice. Because of its subwavelength period, a zeroth order grating does not diffract a normally incident light into non-normal directions; therefore, its diffractive properties are described merely by its transmission and reflection coefficients. When the refractive index contrast between the disconnected scatterers and their surrounding materials are high, the interactions among the scatterers become negligible and the scattering by each individual scatterer is dominated by its own proprieties rather than by a collective coupled response from the entire array. We refer to a high contrast zero order gratings composed of disconnected scatterers which operates in this local scattering regime as periodic high contrast arrays.

The simulated magnetic energy density of the grating shown in Fig. 1 (a) is plotted in in Fig. 2 (b). The simulation is performed using the rigorous coupled wave analysis (RCWA) technique at the wavelength of $\lambda=1550 \mathrm{~nm}$. The post diameters are $400 \mathrm{~nm}$, the lattice constant is $800 \mathrm{~nm}$, and the posts are $940 \mathrm{~nm}$ tall. In Fig. 2 (b), a plane wave with magnetic energy density 1 is incident from the top and the fused silica substrate is located under the posts. As this figure shows, the light is concentrated inside the posts, and each post behaves as a low quality factor resonator. The concentration of the fields inside the aSi posts and the relatively small field amplitudes in the volume between the posts indicate weak coupling among the posts. Since the zeroth order high contrast grating considered here operates in the local scattering regime and exhibit large transmission, we refer to it as a periodic high contrast transmitarray (HCTA).

Aperiodic HCTAs are created by gradually changing the properties of the scatterers of a periodic HCTA while keeping its lattice unchanged. Figure 1 (d) shows an example of a HCTA that is created by gradually changing the post diameters of the periodic HCTA depicted in Fig. 1 (a). Since the posts are weakly coupled to each other, the local transmission and reflection properties in each unit cell of the aperiodic HCTA are dominated by the post located at that unit cell. The main purpose of arranging the posts on a periodic lattice with subwavelength period is to use the interference among the 
optical waves scattered by them to suppress the diffraction into the non-zero diffraction orders. This suppression is more effective when the post diameters vary gradually across the HCTA. The transmission and phase of the transmission coefficient for the periodic HCTA shown in Fig. 1 (a) are computed as a function of the diameter of the posts, and are shown in Fig. 1 (c). As Fig. 1 (c) shows, by proper selection of the diameter of the posts in the $200 \mathrm{~nm}$ to $550 \mathrm{~nm}$ range, large transmission with any arbitrary phase can be achieved. As a result, any gradually varying phase mask can be readily implemented by using an aperiodic HCTA whose posts are selected such that locally impart phase shifts corresponding the desired phase mask.

We designed several HCTA lenses that focus the light emitted from a cleaved single mode fiber. The micro-lenses had the same diameter of $400 \mu \mathrm{m}$, and different focusing distances ranging from $50 \mu \mathrm{m}$ to $500 \mu \mathrm{m}$. We first computed the optimum phase profiles for these lenses using a rigorous method presented in detail in [34]. Then, we sampled the phase profiles at the lattice points of a triangular lattice with the period of $800 \mathrm{~nm}$. Next, we used the relation between the phase of the transmission coefficient and the diameter of the aSi posts (shown in Fig. 1 (c)) to determine the diameter of each posts at its corresponding lattice points.

The high NA lenses were fabricated on a fused silica substrate. First, a $940 \mathrm{~nm}$ layer of aSi was deposited using plasma enhanced chemical vapor deposition at $200^{\circ} \mathrm{C}$. Then, a positive resist was spin coated on top of the aSi layer, and patterned using electron beam lithography. A $70 \mathrm{~nm}$ layer of aluminum oxide was deposited on the resist by electron beam evaporation and was patterned by lifting up the resist in a solvent. The aSi posts were created by dry etching the aSi layer with the patterned aluminum oxide etch mask. Finally, the aluminum oxide mask was etched in a 1:1 mixture of ammonium hydroxide and hydrogen peroxide heated to $80^{\circ} \mathrm{C}$. An optical micrograph of one of the fabricated lenses is shown in Fig. 2 (a), and scanning electron microscope images showing the top and tilted view of the aSi posts are presented in Fig. 2 (b).

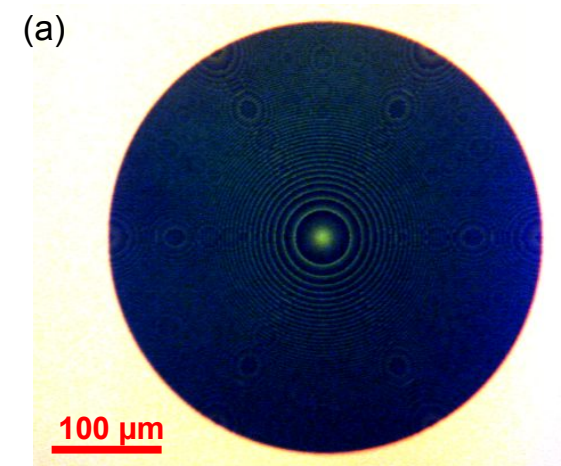

(b)
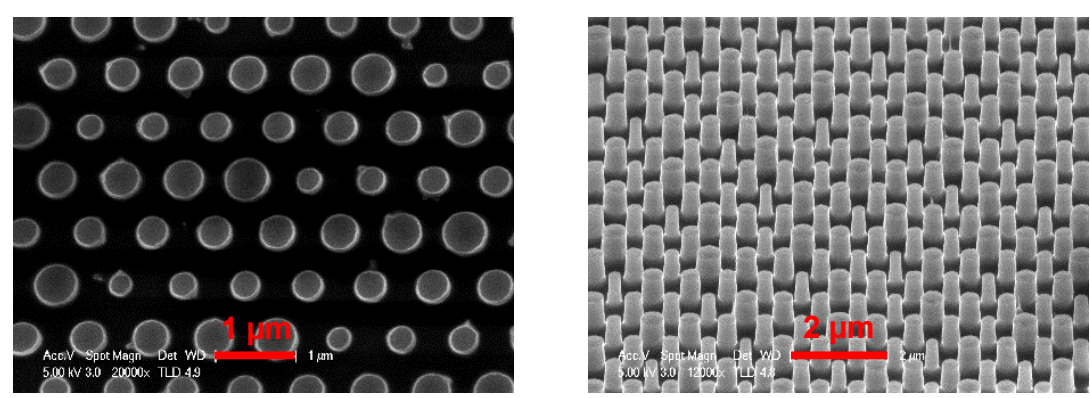

Figure 2. (a) Optical micrograph, and (b) scanning electron microscope images of high NA HCTA micro-lenses. 


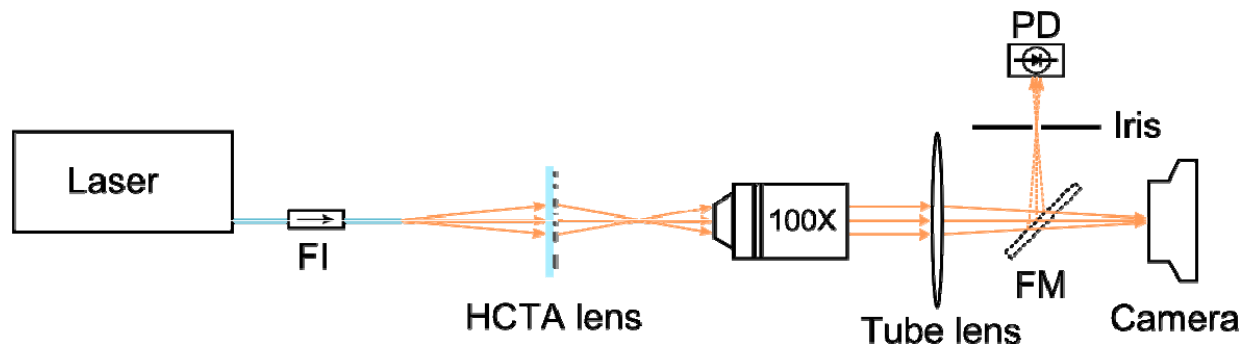

Figure 3. Schematic diagram of the measurement setup used for characterization of the HCTA micro-lenses. PC: polarization controller, FI: fiber isolator, FM: flip mirror, PD: photodetector.

We characterized the fabricated HCTA micro-lenses using the setup schematically shown in Fig. 3. Light from a tunable laser (1450 nm-1590 nm tuning range) was passed through a fiber isolator and emitted from the cleaved tip of a smf$28 \mathrm{e}+$ single mode fiber. The emitted light was focused by a HCTA micro-lens and the plane of focus of the micro-lens was imaged using a custom built microscope composed of a $100 \mathrm{X}$ objective lens and $20 \mathrm{~cm}$ focal length tube lens. A phosphorous coated CCD camera was used to capture the near infrared images. The magnification of the microscope was determined by imaging a sample with known geometrical dimensions.

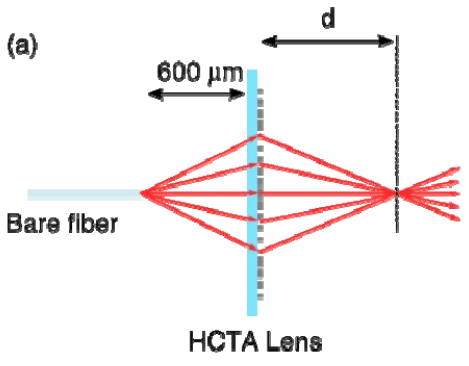

(b)
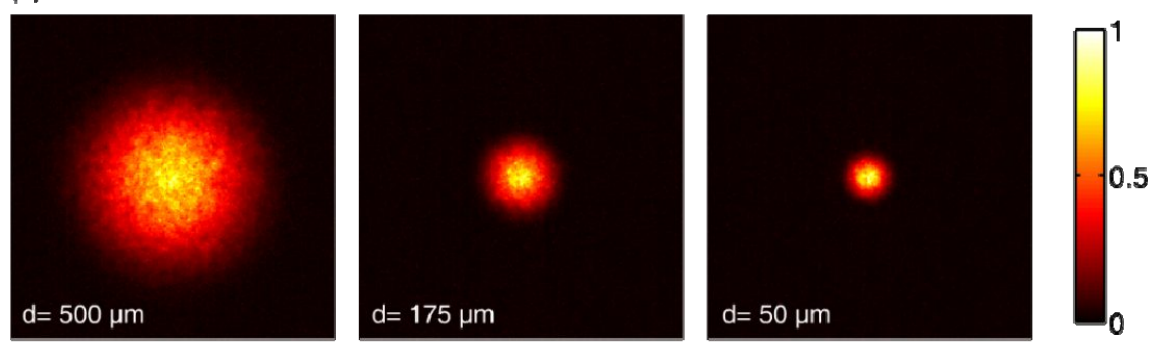

$\underline{2 \mu m}$

(c)
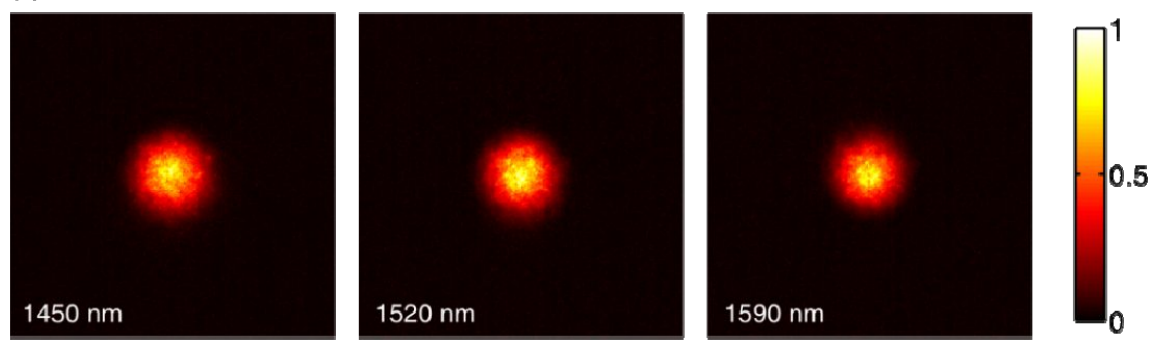

$2 \mu \mathrm{m}$

Figure 4. (a) Schematic illustration of focusing of laser light emitted from a cleave fiber tip by a HCTA micro-lens. (b) Measured focal plane intensity profiles for three high NA lenses with different focusing distances at $\lambda=1550 \mathrm{~nm}$. (c) Focal plane optical intensity profiles for a lens with focusing distance of $175 \mu \mathrm{m}$ recorded at three wavelengths close to the micro-lens design wavelength of $\lambda=1550 \mathrm{~nm}$. 
Recorded focal plane intensity profiles for three micro-lenses with different focusing distances at wavelength of $\lambda=1550$ $\mathrm{nm}$ are shown in Fig. 4 (b). The micro-lenses with smaller focusing distances have higher NA and smaller spot size. We fitted 2D Gaussian functions to the measured intensity profiles shown in Fig. 4 (b), and found FWHM spot sizes of $2.37 \lambda, 0.97 \lambda$, and $0.57 \lambda$ for the micro-lenses with focusing distances of $500 \mu \mathrm{m}, 175 \mu \mathrm{m}$, and $50 \mu \mathrm{m}$, respectively.

To measure the transmitted optical power, we placed a mirror in front of the camera and directed the beam to a photodetector. The transmission efficiency of a micro-lens was determined by dividing the power measured by the photodetector when the micro-lens focused the fiber light and the microscope was imaging the micro-lens' plane of focus by the power measured when the micro-lens was removed from the setup and the objective lens was moved to directly image the fiber tip. We obtained $87 \%, 77 \%$, and $49 \%$ transmission efficiencies for micro-lenses with focusing distances of $500 \mu \mathrm{m}, 175 \mu \mathrm{m}$ and $50 \mu \mathrm{m}$, respectively. To differentiate between the power transmitted through a microlens and the focused power, we put an iris in front of the photodetector and adjusted the radius of the iris to three times the FWHM spot size measured using the camera. We measured focusing efficiencies of $82 \%, 73 \%$, and $42 \%$ for the micro-lenses with focusing distances of $500 \mu \mathrm{m}, 175 \mu \mathrm{m}$, and $50 \mu \mathrm{m}$, respectively. The lower efficiency of the microlenses with smaller focusing distances and higher numerical apertures is due to their faster varying phase profiles which leads to less number of sampling point per $2 \pi$ phase shift [34].

We also characterized the chromatic aberration of the HCTA micro-lenses by measuring their focal spots and focusing efficiencies at different wavelengths. The measured focal plane optical intensities of the micro-lens with focusing distance of $175 \mu \mathrm{m}$ at three different wavelengths are shown in Fig. 4 (c). By fitting 2D Gaussian functions to these intensity profiles, we obtained FWHM spot sizes of $1.2 \lambda, 1.08 \lambda$, and $0.97 \lambda$ and focusing efficiencies of $62 \%, 75 \%$, and $70 \%$ at wavelengths of $1450 \mathrm{~nm}, 1520 \mathrm{~nm}$, and $1590 \mathrm{~nm}$, respectively. The focusing distance of the micro-lens also moved by approximately $20 \mu \mathrm{m}$ per $100 \mathrm{~nm}$ wavelength change.

\section{CONCLUSION}

We experimentally demonstrated flat micro-lenses with subwavelength thickness, focusing efficiencies as large as $82 \%$, and FWHM spot sizes as small as $0.57 \lambda$. We showed that the HCTA platforms can be used to realize efficient high NA micro-lenses because they provide high transmission and weak coupling among scatterers forming the arrays. The weak coupling allows for sampling of the phase profiles with high spatial resolution. Furthermore, the HCTAs not only do not suffer from the fundamental limitations and substantial absorption losses of plasmonic metasurfaces, but they have larger feature sizes and smaller aspect ratio scatterers compared to the low contrast aperiodic gratings operating in the effective medium regime. There is a trade-off between the focusing efficiency and NA of an HCTA micro-lens which is caused by the limited resolution sampling of the phase profiles. Therefore, shrinking the lattice constant of the HCTA might reduce this trade-off. The unmatched efficiency and diffraction limited performance of the HCTA micro-micro-lenses we presented here, combined with their planar structure allow for realization of efficient optical systems on a chip by cascading multiple HCTA based diffractive elements.

\section{REFERENCES}

[1] T. Fujita, H. Nishihara, and J. Koyama, "Blazed gratings and Fresnel lenses fabricated by electron-beam lithography," Opt Lett, 7(12), 578-80 (1982).

[2] T. Shiono, K. Setsune, O. Yamazaki et al., "Rectangular-apertured micro-Fresnel lens arrays fabricated by electron-beam lithography," Appl Opt, 26(3), 587-91 (1987).

[3] T. Shiono, M. Kitagawa, K. Setsune et al., "Reflection micro-Fresnel lenses and their use in an integrated focus sensor," Appl Opt, 28(16), 3434-42 (1989).

[4] M. Haruna, M. Takahashi, K. Wakahayashi et al., "Laser beam lithographed micro-Fresnel lenses," Appl Opt, 29(34), 5120-6 (1990).

[5] L. Y. Lin, S. S. Lee, K. S. J. Pister et al., "Micro-machined three-dimensional micro-optics for integrated freespace optical system," IEEE Photonics Technology Letters, 6(12), 1445-1447 (1994).

[6] B. Morgan, C. M. Waits, J. Krizmanic et al., "Development of a deep silicon phase Fresnel lens using grayscale lithography and deep reactive ion etching," Journal of Microelectromechanical Systems, 13(1), 113-120 (2004). 
[7] L. B. Kong, X. J. Yi, K. Lian et al., "Design and optical performance research of multi-phase diffractive microlens arrays," Journal of Micromechanics and Microengineering, 14(8), 1135-1139 (2004).

[8] W. Stork, N. Streibl, H. Haidner et al., "Artificial distributed-index media fabricated by zero-order gratings," Opt Lett, 16(24), 1921-3 (1991).

[9] F. T. Chen, and H. G. Craighead, "Diffractive phase elements based on two-dimensional artificial dielectrics," Opt Lett, 20(2), 121-3 (1995).

[10] M. E. Warren, R. E. Smith, G. A. Vawter et al., "High-efficiency subwavelength diffractive optical element in GaAs for 975 nm," Opt Lett, 20(12), 1441-3 (1995).

[11] F. T. Chen, and H. G. Craighead, "Diffractive lens fabricated with mostly zeroth-order gratings," Optics Letters, 21(3), 177-179 (1996).

[12] P. Lalanne, S. Astilean, P. Chavel et al., "Blazed binary subwavelength gratings with efficiencies larger than those of conventional échelette gratings," Optics Letters, 23(14), 1081 (1998).

[13] F. M. Huang, T. S. Kao, V. A. Fedotov et al., "Nanohole array as a lens," Nano Lett, 8(8), 2469-72 (2008).

[14] L. Verslegers, P. B. Catrysse, Z. Yu et al., "Planar lenses based on nanoscale slit arrays in a metallic film," Nano Lett, 9(1), 235-8 (2009).

[15] N. Yu, P. Genevet, M. A. Kats et al., "Light propagation with phase discontinuities: generalized laws of reflection and refraction," Science, 334(6054), 333-7 (2011).

[16] P. Genevet, N. F. Yu, F. Aieta et al., "Ultra-thin plasmonic optical vortex plate based on phase discontinuities," Applied Physics Letters, 100(1), 013101 (2012).

[17] F. Aieta, P. Genevet, M. A. Kats et al., "Aberration-free ultrathin flat lenses and axicons at telecom wavelengths based on plasmonic metasurfaces," Nano Lett, 12(9), 4932-6 (2012).

[18] X. J. Ni, A. V. Kildishev, and V. M. Shalaev, "Metasurface holograms for visible light," Nature Communications, 4, (2013).

[19] E. Karimi, S. A. Schulz, I. De Leon et al., "Generating optical orbital angular momentum at visible wavelengths using a plasmonic metasurface," Light-Science \& Applications, 3, e167 (2014).

[20] D. Fattal, J. J. Li, Z. Peng et al., "Flat dielectric grating reflectors with focusing abilities," Nature Photonics, 4(7), 466-470 (2010).

[21] F. Lu, F. G. Sedgwick, V. Karagodsky et al., "Planar high-numerical-aperture low-loss focusing reflectors and lenses using subwavelength high contrast gratings," Opt Express, 18(12), 12606-14 (2010).

[22] Z. Peng, D. A. Fattal, A. Faraon et al., "Reflective silicon binary diffraction grating for visible wavelengths," Opt Lett, 36(8), 1515-7 (2011).

[23] A. B. Klemm, D. Stellinga, E. R. Martins et al., "Experimental high numerical aperture focusing with high contrast gratings," Opt Lett, 38(17), 3410-3 (2013).

[24] Y. Chiu, C. H. Huang, and Y. C. Hsu, "High numerical-aperture microlens fabricated by focused ion beam milling - art. no. 66200L," Optical Data Storage 2007, 6620, L6200-L6200 (2007).

[25] A. V. Kildishev, A. Boltasseva, and V. M. Shalaev, "Planar photonics with metasurfaces," Science, 339(6125), 1232009 (2013).

[26] N. Yu, and F. Capasso, "Flat optics with designer metasurfaces," Nat Mater, 13(2), 139-50 (2014).

[27] F. Monticone, N. M. Estakhri, and A. Alu, "Full control of nanoscale optical transmission with a composite metascreen," Phys Rev Lett, 110(20), 203903 (2013).

[28] A. Arbabi, and A. Faraon, "Fundamental Limits of Ultrathin Metasurfaces," arXiv preprint arXiv:1411.2537, (2014).

[29] W. B. Chen, and Q. W. Zhan, "Three-dimensional focus shaping with cylindrical vector beams," Optics Communications, 265(2), 411-417 (2006).

[30] D. Lin, P. Fan, E. Hasman et al., "Dielectric gradient metasurface optical elements," Science, 345(6194), 298302 (2014).

[31] A. Arbabi, M. Bagheri, A. J. Ball et al., [Controlling the Phase Front of Optical Fiber Beams using High Contrast Metastructures - OSA Technical Digest (online)] Optical Society of America, San Jose, California(2014).

[32] S. Vo, D. Fattal, W. V. Sorin et al., "Sub-Wavelength Grating Lenses With a Twist," Ieee Photonics Technology Letters, 26(13), 1375-1378 (2014).

[33] A. Arbabi, Y. Horie, and A. Faraon, [Planar Retroreflector - OSA Technical Digest (online)] Optical Society of America, San Jose, California(2014). 
[34] A. Arbabi, Y. Horie, A. J. Ball et al., "Subwavelength-thick Lenses with High Numerical Apertures and Large Efficiency Based on High Contrast Transmitarrays," arXiv preprint arXiv:1410.8261, (2014). 